\title{
Platelet Size in Thrombocytosis
}

Galveston, Tex.

J.D. Bessman, MD, Division of Hematology-Oncology, The University of Texas Medical Branch at Galveston, Galveston, TX 77550 (USA)

Levin, J.; Bessman, J.D.: The inverse relation of platelet count and size. Studies in hematologic disorders and evidence that platelet size is not related to platelet age. J. Lab. clin. Med. 101: 297-309(1983)

Rowan, R.M.; Fraser, C: Platelet size distribution analysis; in England, van Assendelft, Advances in haematological methods: The blood count, (CRC Press, Boca Raton 1982).

Bessman, J.D.; Williams, L.J.; Gilmer, P.R.: Mean platelet volume. Am. J. clin. Path. 76: 289-293(1981).

Bessman, J.D.: The relation of megakaryocyte ploidy and platelet size. Am. J. Hematol. (in press, 1984).

Robbins and Barnard [1] show that in reactive 2 thrombocytosis mean platelet volume (MPV) and platelet count vary inversely. We have shown this inverse relation in surgical patients with sepsis [2] and 3 in subjects with pneumonia [3]; figure 2 of Robbins and Barnard is an excellent independent confirmation. This inverse relation appears to be an extension of the inverse relation of platelet count and MPV that is seen in normal subjects [4, 5]. MPV correlates di- 4 rectly with megakaryocyte DNA content (ploidy), and megakaryocyte ploidy is reduced in reactive thrombocytosis [6]. Thus, reduced MPV in reactive thrombocytosis reflects an alteration in megakaryo- 5 cytopoiesis - more megakaryocytes, less endoredu-plication - and is an extension of whatever causes the inverse relation of platelet count and size in normals. 6 Expression of a particular patient's sequential platelet count and MPV, as thrombocytosis develops and remits, against the background of the nomogram of normal subjects $[2,3,5]$ would highlight the nonlinear inverse relation.

References

1 Robbins, G.; Barnard, D.L.: Thrombocytosis and microthrombocytosis: a clinical evaluation of 372 cases. Acta haemat. 70: 175-182(1983). 